\title{
Arquitectura Sónica de Pulsación digital: Diseño interdisciplinar paramétrico y fabricación digital aplicada a instrumentos musicales e instalaciones sonoras
}

\author{
Eric Goldemberg \\ Florida International University \\ goldembe@fiu.edu
}

\begin{abstract}
This paper proposes to demonstrate the capacity of parametric design and digital fabrication as catalysts for new sensorial experiences, in the context of an approach to the field of experimental design of body architectures. Projects thate architecture and music by means of technological innovations offer new possibilities for artistic productions therefore a new model of interdisciplinar integration is proposed through this investigation. Through the analysis of this kind of work, new fields of design can be teased out and proposed as manifestations of the expanded field of architecture.
\end{abstract}

Keywords: Interdisciplinar; 3D-print; música; arquitectura; paramétrico.

\section{Introducción}

Esta presentación se propone demostrar el potencial del diseño paramétrico y la fabricación digital como catalizadores de nuevas experiencias sensoriales, en el marco de una aproximación al campo experimental del diseño de arquitecturas aplicadas al cuerpo humano. Una lectura detenida del diseño contemporáneo guiado por lo digital revela sensaciones que gozan con los ritmos pulsantes en la articulación de superficies en arquitectura, energizadas por el ritmo surgido de una creciente conciencia del detalle dentro de una sensibilidad de tectónica topológica; efectos rítmicos acentúan la imagen residual de ornamentos detallados como huella, un índice de actividad registrado sobre membranas arquitectónicas que codifican transformación espacial y diferencia. Debido a la amplia capacidad de transmutación de escalas proyectuales en el campo virtual, estas mismas sensaciones se manifiestan en el diseño de objetos a escala del cuerpo humano con mayor intensidad aun, alterando inclusive la percepción de las proporciones del cuerpo modificado por prótesis de alta calidad estética.

\section{Metodología}

Se utilizara como evidencia una serie de proyectos desarrollados por un equipo inter-disciplinar integrado por arquitectos, músicos, lutiers y compositores. Este equipo del cual el autor forma parte - enriquecido por la hibridación colaborativa proveniente de los conocimientos expertos y las capacidades de ejecución artesanal de variadas procedencias, se ha abocado a la investigación y reelaboración de la relación ergonómica que se establece entre los músicos y los instrumentos musicales por medio de técnicas de mapeado digital de las posturas durante la performance, y la consecuente re-concepción de la estructura prostética de dichos artefactos sonoros y su estética emergente. El modelado en 3D permite una optimización de los puntos de fricción entre el cuerpo y el artefacto, de modo tal que se descubren nuevas capacidades para la producción de sonido, utilizando geometrías tridimensionales maleables y nuevos materiales de impresión digital como el plástico PLA. Para entender al alcance cultural profundo de este tipo de practica es menester situar un marco conceptual donde se inscriben los proyectos desarrollados en forma trans-disciplinar, unificados por el concepto de pulsation y su manifestación en las formas rítmicas, generadas digitalmente.

\section{Las singularidades del efecto rítmico}

"Según Lyotard en un ritmo dado, la condición para repetir identidad formal y regularidad - debe de algún modo ser establecida en una matriz cuyo propósito es colapsar dichas regularidades y aplastar dichas identidades en su propio camino hacia la 'mala forma'. El ritmo entonces, compuesto tanto de extinción como de repetición, es la forma de esta 'mala forma'. Es la violencia quieta en espera de forma, así como es la forma de la violencia. Dentro del 'gran arte', la forma es construida para resguardarse de la violencia de este ritmo, para conseguir la permanencia de la configuración, su inmunidad al asalto. Por el contrario, es en las formas culturales más bajas y vulgares donde lo visual es diariamente invadido por lo 'pulsátil': las luces parpadeantes de los carteles de neón, los 'flipa booms' a través de los cuales lo visualmente inerte es impulsado hacia lo sugestivamente obsceno, el efecto estroboscópico de las máquinas de pinball y videojuegos -y todo esto unido por el insistente ritmo de la música de rock a través de los parlantes del auto o filtrándose sin voz a través de auriculares." (Rosalind Krauss, 1997, p.164).

Pulsation sitúa la discusión de las prácticas arquitectónicas y de diseño de objetos que hacen uso extensivo de la capacidad operacional del diseño digital para develar cualidades afecto-perceptivas del espacio mediante la articulación rítmica. Hay un cambio de paradigma en la percepción espacial debida al intenso uso de técnicas computacionales en arquitectura y la posibilidad de procesar y manipular cantidades de datos masivas, donde el ritmo es ahora percibido como jugando un rol activo en la formación 


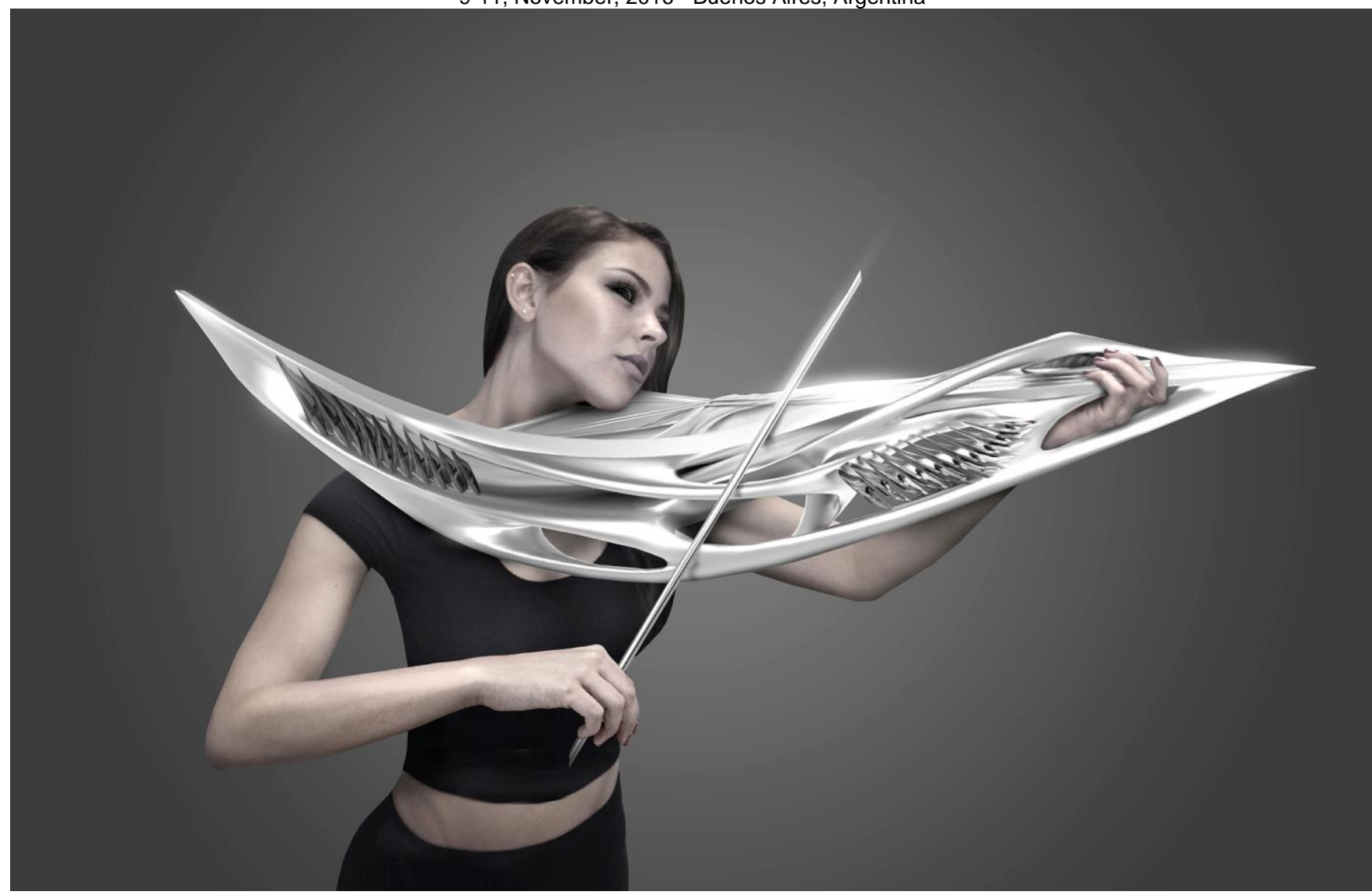

Figura 1: 2-string piezoelectric violin - adaptacion ergonómica a la postura de performance facilitada por el diseño en 3D y la impresión digital MONAD Studio | Eric Goldemberg + Veronica Zalcberg, en colaboración con el lutier Scott F. Hall

del espacio y la articulación tectónica, reclamando el primer plano en el campo de la figuración y no sólo incrustado o indexado en la estructura. La actividad pulsante que resulta del diseño digital intenso no es sólo revelada a través de constricciones estructurales o programáticas sino que está ahora tomando una presencia mucho más importante en la articulación de la topología de los diseños, creando tramas

que operan para articular transiciones entre las diversas escalas de representación, re-vigorizando la capacidad del ornamento para dar carácter y fomentar estados de ánimo, ambiente y atmósfera a través del lente modificado y afinado de la percepción espacial y sus afiliaciones afectivas.

\section{Resultados}

Las formas emergentes de estos instrumentos musicales innovadores observan relaciones de proporciones y funcionalidad que respetan la tipología histórico-funcional que da origen a los violines, guitarras, cellos e instrumentos de viento concebidos ahora en forma paramétrica; pero su morfología y su estética resultante como así también su capacidad sónica producen artefactos radicalmente novedosos que incitan a nuevas producciones artísticas. El proceso inherente de construcción de los instrumentos por medio de impresoras de $3 d$ resulta en una materialización que genera sorprendentes cualidades sonoras ya que la impresora digital construye una estructura interna de soportes que generan múltiples cavidades hexagonales y estas transmiten frecuencias de sonido que producen efectos novedosos (ver imágenes adjuntas).

\section{Piezoelectricidad}

Algunos materiales, tales como cristales de cuarzo y ciertas cerámicas, son naturalmente piezoeléctricos. Esto significa que cuando el material se somete a algún tipo de estrés o presión, los átomos en la red de material se reorganizan ligeramente a sí mismos, dando como resultado un exceso de carga que se acumula en las zonas opuestas del material. Cualquier separación de carga eléctrica crea una tensión que puede formar parte de un circuito eléctrico, y tiene un sensor de presión eléctrica básica. Lo contrario también funciona. Enviar una corriente a través de un material piezoeléctrico y el material será "exprimir sí", vibrando su estructura cristalina atómica en respuesta a la corriente.

El efecto piezoeléctrico fue descubierto por primera vez en 1880 por Pierre Curie (esposo de Marie Curie) y su hermano, Jacques Curie, y se utiliza hoy en día en todo, desde el escaneo de microscopios de sonda a las fuentes de encendido de barbacoas de gas propano y encendedores (presión de los dedos provoca una señal eléctrica arco que 


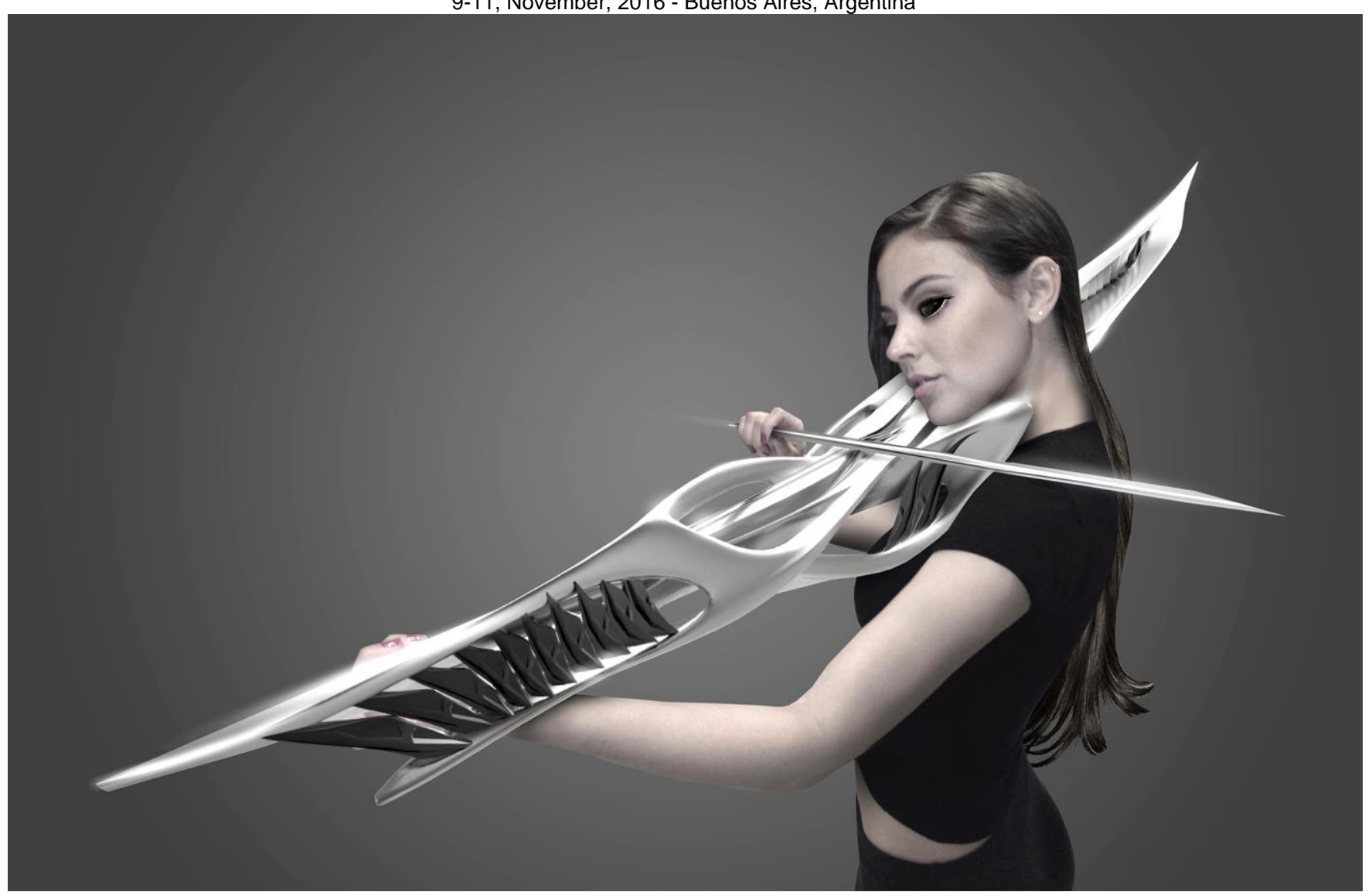

Figura 2: 2-string piezoelectric violin - desarrollo de geometrías curvilíneas en 3D por medio variaciones rítmicas curvilíneas

MONAD Studio | Eric Goldemberg + Veronica Zalcberg, en colaboración con el lutier Scott F. Hall

enciende el combustible). La piezoelectricidad se utiliza también en los detectores de sonido sensibles, ya que las vibraciones de un material piezoeléctrico se pueden traducir en un sonido eléctrico.

Esto nos lleva a los violines. Muchos músicos acústicos ya utilizan pastillas piezoeléctricas, pequeños "micrófonos" que se montan en el puente de un violín con el fin de transformar las vibraciones del violín en un sonido eléctrico para su

También se han diseñado y fabricado instalaciones sonoras incluyendo un mural que aloja a dos guitarras eléctricas de diseño y fabricación digital encastradas en una estructura de 6 metros de largo por 2 metros de alto construida por procesos de CAD-CAM (ver imagen superior adjunta). La topografía tridimensional del mural es activada por medio de dispositivos manuales transductores (micro-speakers) que emiten sonidos pre-compuestos digitalmente sobre dichas superficies. La composición sonora ambiental resultante es producto de la reacción interactiva que el performer/musician desarrolla deslizando el transductor sobre las inflexiones geométricas de las superficies del mural.

\section{Conclusiones}

Estos proyectos de integración experimental entre arquitectura y música por medio de la tecnología de diseño y posterior amplificación o transformación.

El trabajo de MONAD Studio va un paso más allá. Su violín de dos cuerdas parece funcionar únicamente por el efecto piezoeléctrico; es posible imaginar el músico presionando sus dedos y el arco en áreas específicas, piezoeléctricos del violín, lo que enviaría una corriente a través de un amplificador y altavoz para crear música. es probable que su sonido sea mucho más eléctrico que el de un violín tradicional.

fabricación digital presentan novedosas posibilidades para producciones artísticas y se propone por medio de este trabajo, un modelo de integración interdisciplinar donde las distintas disciplinas pueden acoplarse y producir resultados que cuestionen modelos existentes y abran campos de investigación que extienden los limites de la arquitectura al campo expandido del conocimiento espacial-sensorial; es por medio de la interacción multidisciplinar y la integración de plataformas digitales y tradiciones artesanales de construcción que se pueden obtener innovaciones profundas. La arquitectura de pulsation celebra la duración, mejora nuestra conciencia acerca del pasaje del tiempo indexado en la forma; para Bergson la duración es el continuo progreso del pasado que roe hacia el futuro en el cual se expande a medida que avanza. La duración involucra un proceso de repetición y diferencia, es irreversible ya que la conciencia no puede atravesar el mismo estado dos 

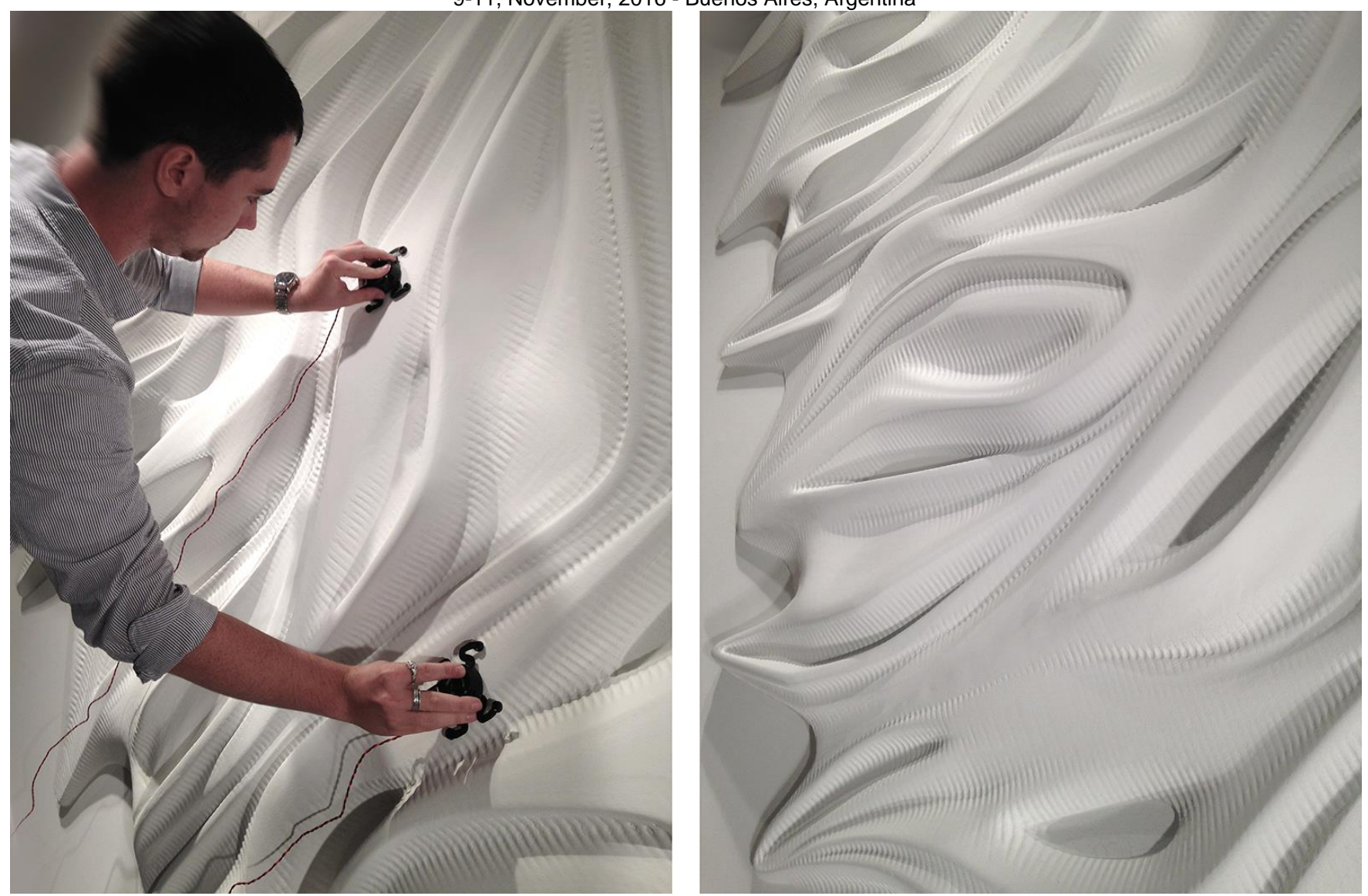

Figura 3: Abyecto Sonic environment - Topologia sónica interactiva activada por transductores manuales, improvisacion digital proyectada.

MONAD Studio | Eric Goldemberg + Veronica Zalcberg, en colaboración con el lutier Scott F. Hall y compositor Jacob Sudol

veces; no somos capaces de vivir y revivir un único momento. La noción de duración está embebida de rítmica, palpitación, estrategias de vibración para la articulación de membranas que extienden las cualidades tectónicas a la experiencia espacial; una multitud de componentes sincronizados que irradian micro alianzas entre partes, distribuyendo tramas ornamentales que dan carácter y atmósfera a la arquitectura. Esta modalidad de diseño basada en el feedback activo que se produce entre el ritmo de la música y las características formales rítmicas de los instrumentos impresos digitalmente y las instalaciones sonoras define un nuevo tipo de performance artística que se materializa en forma novedosa de acuerdo a las nuevas capacidades tecnológicas de materialización, señalando el camino a seguir para la innovación en las artes y la arquitectura. Propone un entendimiento novedoso de la arquitectura basado en el potencial del diseño digital para superar su comúnmente aceptado rol instrumental, demostrando el potencial de involucrarse en temas más profundos e inclusivos de otras artes para dar vigor a un discurso de relaciones parte-todo a través de la lente de la sensación rítmica y su expansión trans-disciplinar.

\section{Referencias}

Lynn, G. (1992). Animate Form. New York, NY: Princeton Architectural Press.

Merleau-Ponty, M. (1962). Trans: Colin Smith. Phenomenology of Perception London: Routledge \& Kegan Paul

Bogue, R., (2003). Musica naturans: Deterritorializing the Refrain. In R. Bogue, Deleuze on Music, Painting and the Arts (pp. 16-18). New York, NY and London: Routledge.

Krauss, R., (1997), Pulse. In Y.A. Bois and R. E. Krauss, Formless: A User's Guide (pp 164). New York, NY: Zone Books

Santa Lucia, A., (2015), Feedbackism. In A. Schieda, Documento Arquis de Arquitectura y Urbanismo : Universo Parametrico (pp 22). Buenos Aires: Universidad de Palermo

Maciel, T.,(2015). The Science of a Piezoelectric Violin. Physics Central. Retrieved

from http://physicsbuzz.physicscentral.com/2015/03/the-science-ofpiezoelectric-violin.html 


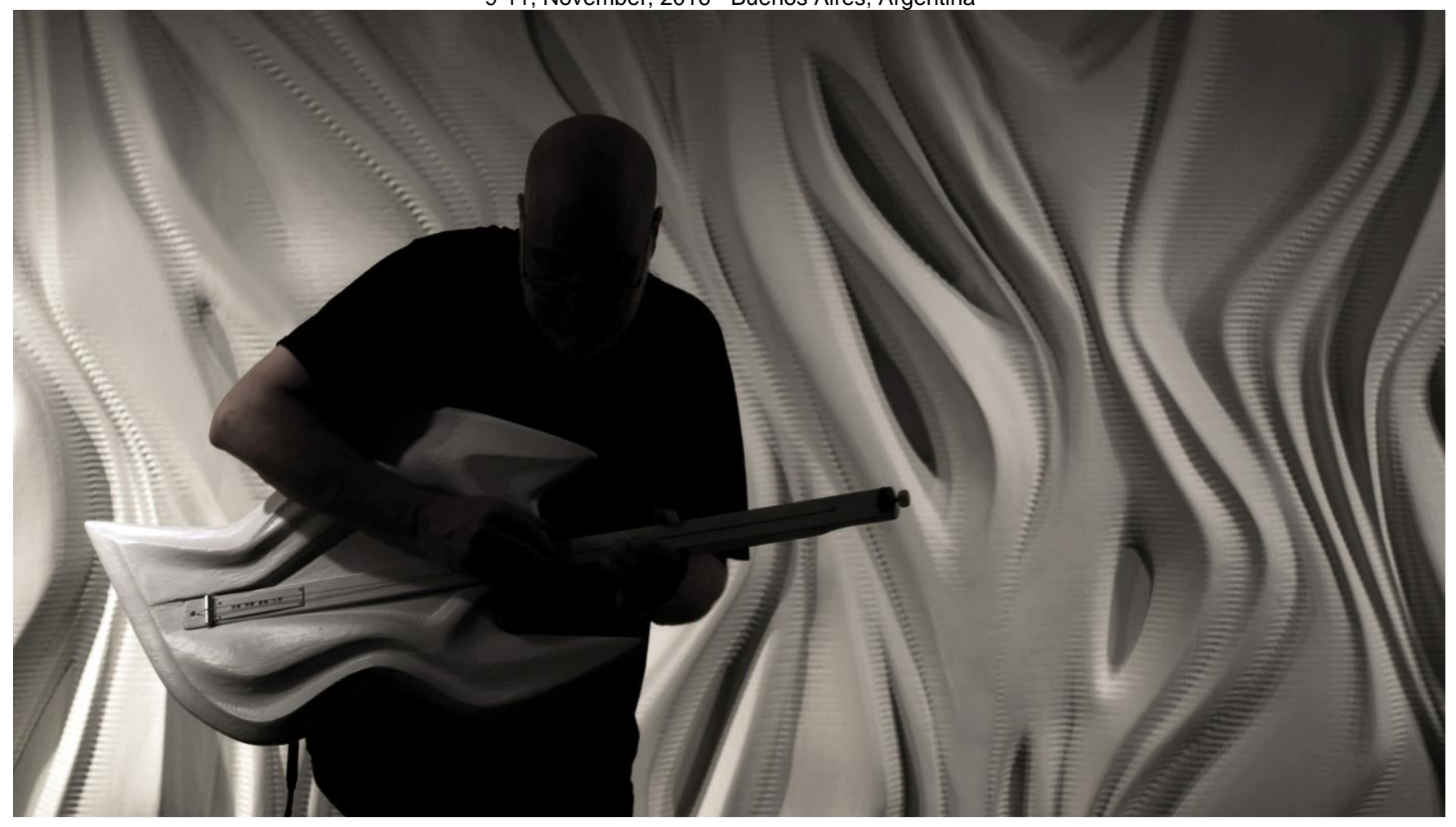

Figura 4: Abyecto Sonic environment - Guitarra fabricada con tecnología digital de CNC, integrada al mural y desmontable para performance MONAD Studio | Eric Goldemberg + Veronica Zalcberg, en colaboración con el lutier Scott F. Hall y compositor Jacob Sudol

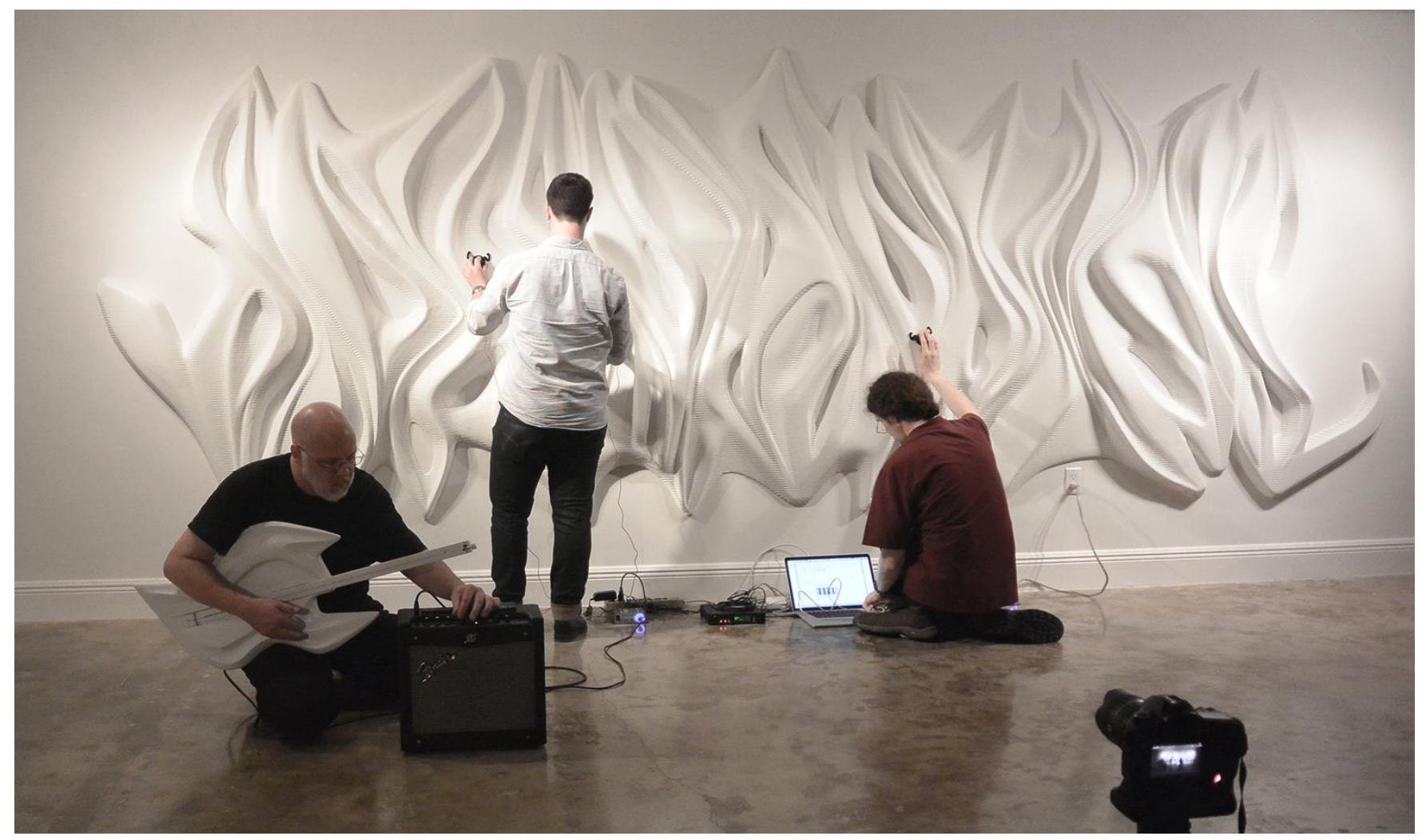

Figura 3: Abyecto Sonic environment - Integracion de distintas formas de interactividad performativa de sónidos análogos y digitales. MONAD Studio | Eric Goldemberg + Veronica Zalcberg, en colaboración con el lutier Scott F. Hall y compositor Jacob Sudol 
SIGraDi 2016, XX Congreso de la Sociedad Ibero-americana de Gráfica Digital 9-11, November, 2016 - Buenos Aires, Argentina

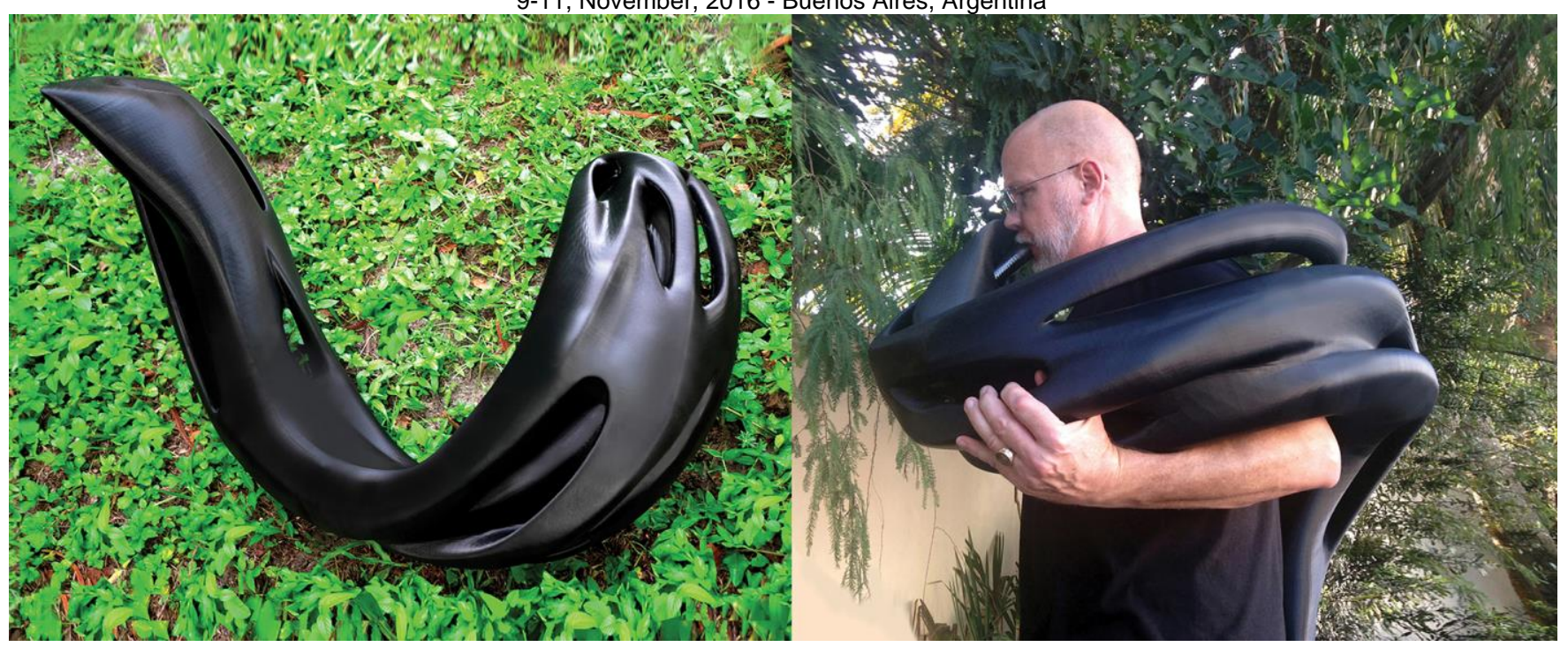

Figura 6: Hornucopian dronepipe - Instrumento de viento diseñado para envolver al performer en una columna de sonido envolvente MONAD Studio | Eric Goldemberg + Veronica Zalcberg, en colaboración con el lutier Scott F. Hall y compositor Jacob Sudol

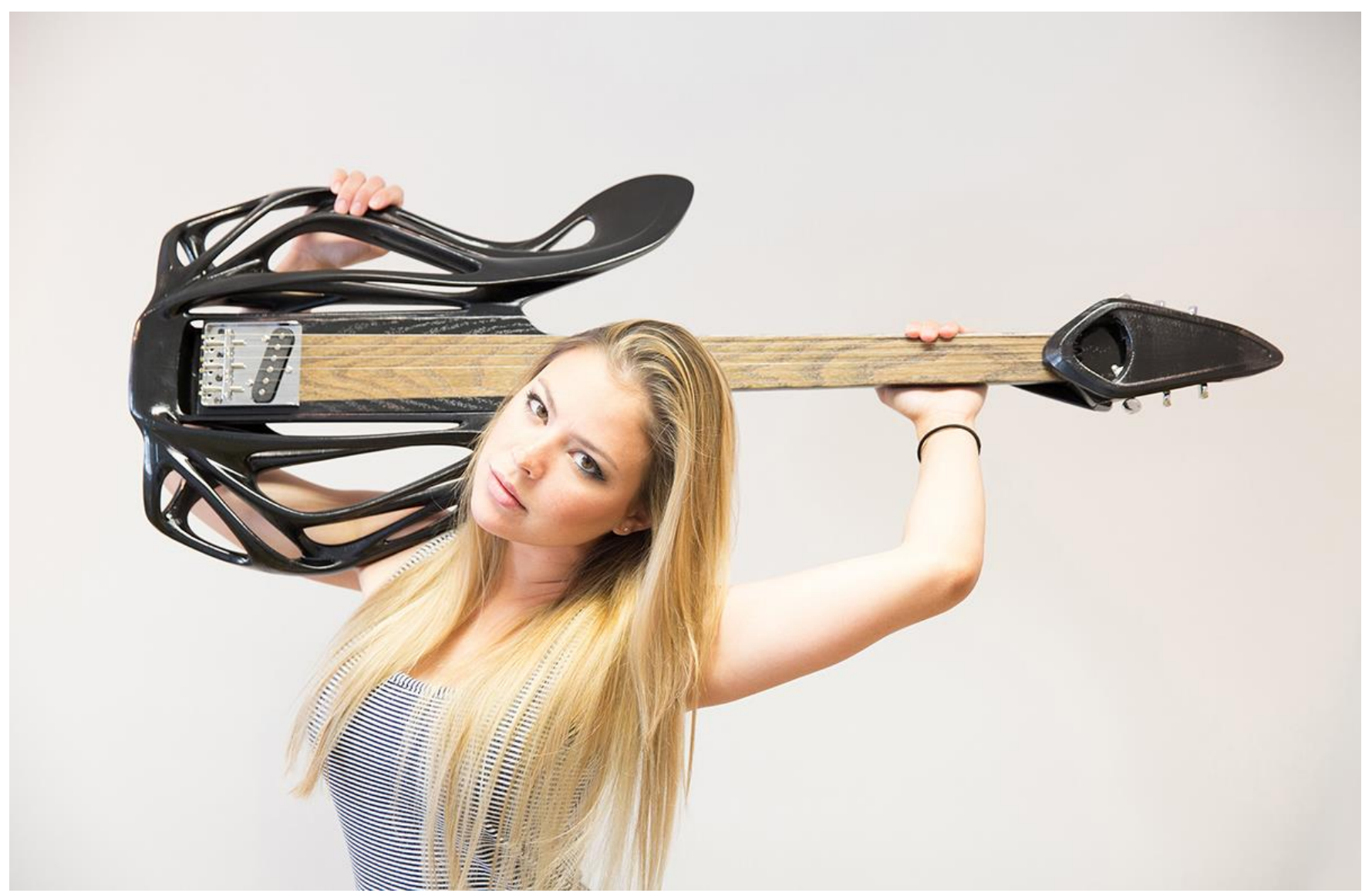

Figura 7: Hybrid Slide guitar - Guitarra eléctrica desarrollada para alta performance en cuanto a su liviandad y transparencia de volumen MONAD Studio | Eric Goldemberg + Veronica Zalcberg, en colaboración con el lutier Scott F. Hall y compositor Jacob Sudol 